FIVE years ago few biologists would have predicted that the structural organisation of eukaryotic genes would be known in such great detail. However, although we now know the primary structure of several genes, the mechanisms by which their transcription is controlled remain unclear. Speakers at the fifteenth Harden conference* set out to bridge this gap between our knowledge of structure and its relation to function.

One system which we are on the way to understanding is the transcription and processing of yeast tRNA genes. The primary transcript of these genes is trimmed at both $5^{\prime}$ and $3^{\prime}$ ends and a short intervening sequence is removed to produce the mature tRNA. J. Abelson (University of California, San Diego) reported work in progress to purify the enzymes responsible for splicing out the intervening sequence, using as a substrate precursors purified from a temperaturesensitive mutant strain deficient in processing. A specific endonuclease and ligase have been separated, which are unusual in that they respectively create and rejoin a $3^{\prime}$ terminal phosphate and a $5^{\prime}$ hydroxy group. E. de Robertis (MRC Molecular Biology Laboratory, Cambridge) reminded us that tRNA precursors microinjected into Xenopus oocytes can be completely processed (Nature 278, 137: 1979). That toad enzymes can correctly splice out the yeast tRNA intervening sequence is surprising since it has diverged markedly in sequence from that of Xenopus. B. Hall (University of Washington, Seattle) has taken advantage of the ability of Xenopus enzymes to process yeast tRNA correctly to probe the relationship between structure and function, by studying the transcription and processing of cloned mutant tRNAs in a Xenopus cell-free system. Twenty mutants selected by loss of nonsense suppressor activity, have been cloned and sequenced. Interestingly, all were found to lie within the $5^{\prime}$ and $3^{\prime}$ limits of the mature tRNA. One mutant, located in the intervening sequence, was not correctly transcribed and gave a primary transcript

- Held at Wye College, Ashford, Kent UK on 16-21 September 1979. and organised by Professor Peter Walker on behalf of the Biochernical Society.

dull, colours are needed to signal a warning, (although there is no evidence that predators learn faster to avoid bright colours). There is also a possible problem in explaining how the genes for warning colour spread in the first place, since each predator has to attack, and perhaps kill, a few individuals to learn its lesson.

Baker and Parker also suggest that some bird colours are used as 'flash colouration'. These are colours of otherwise cryptic animals which are exposed suddenly when a predator attacks. The predator is tem-

\section{Structure and function of eukaryotic genes}

\author{
by Jonathan Wolfe
}

only 48 nucleotides long.

This type of 'surrogate' genetics can clearly provide information about the influence of primary structure on transcription and processing; however results obtained in heterologous systems should be interpreted cautiously. J. Beggs (Plant Breeding Institute, Cambridge) showed us that not all yeast and vertebrate systems are mutually compatible. She transformed yeast with a plasmid carrying the rabbit globin genes and flanking sequences. Globin transcripts were made: however the yeast did not splice out the first intervening sequence and transcription terminated within the second. This point was also made by $C$. Weissmann, in the Harden Lecture, who stressed the value of studying transcription and translation in a homologous system.

The yeast tRNA genes were not the sole topic of the meeting. C. Henschel (University of Zurich) reported results from M. Birnstiel's group who have now almost completely sequenced the sea urchin histone genes. They have found a Hogness box, the putative eukaryotic promoter, a short distance upstream of the $\mathrm{H} 2 \mathrm{~A}$ gene and have constructed a number of plasmids carrying small deletion mutants of this box or of the surrounding sequences. Sea urchin DNA supercoils, removed from the plasmid vector, were then tested (in Xenopus oocytes) for their ability to be transcribed and translated. Deletion of sequences between the Hogness box and the AUG codon of the H2A mRNA was found to have little effect on the amount of transcription. However, removal of the Hogness box greatly reduced transcription and led to variation in the point of initiation. What, at first sight, were contradictory results were
Bendig and $\mathrm{W}$. Folk of the University related by $\mathrm{R}$. Kamen (Imperial Cancer Research Fund, London) reporting work with a deletion mutant isolated by $\mathbf{M}$. of Michigan. He told us that deletion of an apparent Hogness box upsteam of the polyoma virus early region genes actually led to an increase in the rate of transcription initiated at a new site upstream of the deletion. However, the deletion extended into the 5 ' end of the message and may have decreased the rate at which it was translated. One possible explanation of this result was therefore that the early region is under feedback control exercised by one of the protein products and the reduction in this inhibition may more than cancel out any possible decrease in transcription as a result of deletion of the promoter.

Surrogate genetics is clearly a powerful tool to investigate structure-function relationships in straightforward systems but it is of limited value when probing more complex situations. However, we were provided with one example of the rapid progress which molecular biologists can make when working with a complex system already well-characterised genetically. K. Nasmyth (University of Washington, Seattle) reported work in which he has confirmed the geneticists' 'cassette'model of yeast mating type switching. This model explains the 'switch' in mating type from $\alpha$ to a or vice versa which can occur in budding yeasts, by proposing that silent copies of the two mating type genes exist elsewhere in the genome (at loci HML $\alpha$ and HMRa) and that they can reprogramme the locus at which the active mating type is expressed (MAT). Nasmyth has now shown common sequences at the MAT locus and at the loci HML $\alpha$ and HMRa. Clones were selected for their ability to complement MAT $\alpha$ yeast, which are unable to respond to mating type a hormones and it was then straightforward to clone all three loci. Heteroduplex mapping showed that although the clones had sequences in common they contained a or $\alpha$-specific sequences and also sequences specific to each locus.

Jonathan Wolfe is an Assistant Editor of Nature. porarily startled or confused, allowing its potential victim to escape. The bright red eye and black and white head of the night heron (Nycticorax nycticorax) might be such an example.

The evidence adduced by Baker and Parker for their hypothesis consists of a comparative survey of 516 species of western Palaearctic birds. For each species they coded conspicuousness on a scale of 0-5 for different regions of the body crown, head, neck, back, belly and so on. They then carried out a stepwise multiple regression to look for correlations between conspicuousness of each body region and various ecological and life history variables such as diet, gregariousness, mating system, migration distance, and nesting behaviour. The analysis was done separately for adults, juveniles, males, females, summer and winter plumage. The authors admit that there are many inadequacies in their analysis, not least of which is deciding how conspicuous a bird is in its natural habitat.

I can mention only a few of the many 